\section{Radiologische Altersbestimmung}

\section{Rechtliche Zulässigkeit bei hinreichender Beachtung von Maßgaben}

\section{Einführung}

In verschiedenen behördlichen und strafrechtlichen Verfahren muss das Lebensalter von Personen festgestellt werden, um diese Verfahren rechtssicher durchführen zu können. ${ }^{1}$ Ärztliche Untersuchungen zur Feststellung des Alters von Flüchtlingen können auf Grundlage von $\S 49$ Abs. 3 und 6 Aufenthaltsgesetz (AufenthG) und $\S 42$ Abs. 2 f. SGB VIII angeordnet werden. ${ }^{2}$ Ein bundeseinheitliches Verfahren zur Altersfeststellung ist dazu nicht verbindlich vorgegeben; dementsprechend wird das Lebensalter bundesweit auf unterschiedlichen Wegen bestimmt. Bei Vorliegen einer hinreichenden Ermächtigungsgrundlage für Röntgenuntersuchungen ohne medizinische Indikation empfiehlt die internationale und interdisziplinäre Arbeitsgemeinschaft für Forensische Altersdiagnostik (AGFAD) für Altersbegutachtungen bei Jugendlichen und jungen Erwachsenen die Kombination einer körperlichen Untersuchung mit Anamneseerhebung, einer Röntgenuntersuchung der Hand und einer zahnärztlichen Untersuchung mit Anfertigung

1 Zur Altersbestimmung im Strafprozess siehe z. B. Wenske, in Münchener Kommentar zur StPO, 1. Aufl. 2016, § 267 Rn. 254; BGH, Urt. v. 27.08.1997, Az.: 3 StR 331/97, NStZ 1998, 50; zur Altersbestimmung im Ausländerrecht Bruns, in: Hofmann, Ausländerrecht, 2. Aufl. 2016, Rn. 16; zur Altersbestimmung im Familienrecht Dürbeck, Unbegleitete Minderjährige im Familienrecht, FamRZ 2018, 553; OLG Hamm, Beschl. v. 13.03.2006, Az.: 4 UF 35/ 06, BeckRS 2009, 9616 zur Altersbestimmung bei der Anordnung einer Vormundschaft.

2 Siehe dazu den Sachstandsbericht der Wissenschaftlichen Dienste des Deutschen Bundestags „Methoden zur forensischen Altersdiagnostik“ vom 25.1.2018 und zum Thema „Gesetzliche Grundlagen einer Untersuchung zur Altersbestimmung“ vom 22.01.2018 sowie die Ausarbeitung der Wissenschaftlichen Dienste des Deutschen Bundestags zum Thema „Zuständigkeitsfragen zur Altersbestimmung bei minderjährigen Ausländern und zum Eintritt der Volljährigkeit“ vom 17.2.2016; Schmeling/Dettmeyer/Rudolf/Vieth/Geserick, Forensische Altersdiagnostik. Methoden, Aussagesicherheit, Rechtsfragen, Deutsches Ärzteblatt 2016, 113. Flüchtlingen, KJ 2015, 405. eines Orthopantomogramms (Panoramaröntgenaufnahme der Kieferregion). ${ }^{3}$

Umstritten war in den letzten Jahren vor allem die Altersdiagnostik bei Flüchtlingen, deren Angabe, minderjährig zu sein, im Einzelfall zweifelhaft sein kann. Grund dafür kann sein, dass den Betroffenen ihr Geburtstag tatsächlich unbekannt ist und sie sich für minderjährig halten oder dass falsche Angaben in der nachvollziehbaren Hoffnung gemacht werden, Abschiebungen zu erschweren oder Eltern nach Deutschland zu holen. Jugendämter, aber auch das Bundesamt für Migration und Flüchtlinge (BAMF), veranlassen deshalb unter anderem auch radiologische Untersuchungen zur Altersfeststellung. ${ }^{4}$ Deren Zulässigkeit wird zwar gelegentlich angezweifelt. Die Rechtsprechung teilt diese Bedenken jedoch nicht, wenn und soweit die rechtlichen Anforderungen für diese Altersdiagnostik, insbesondere der Grundsatz der Verhältnismäßigkeit, hinreichende Beachtung finden.

Grundlage für die Beauftragung medizinischer Altersgutachten bei Flüchtlingen durch das BAMF sind das Asylgesetz und die Richtlinie 2013/32 EU zu gemeinsamen Verfahren für die Zuerkennung und Aberkennung des internationalen Schutzes, die sogenannte Verfahrensrichtlinie. Nach § 24 Abs. 1 S. 1 AsylG hat das BAMF den Sachverhalt in einem Asylverfahren aufzuklären und die erforderlichen Beweise zu erheben habe. Insoweit besteht auch die Pflicht, sich über verfahrensrelevante Tatsachen in eigener Zuständigkeit Gewissheit zu verschaffen. Hierzu zählt auch die Aufklärung

3 Siehe dazu die im Internet veröffentlichte Abhandlung von Schmeling, Die aktuelle medizinethische Debatte über forensische Altersdiagnostik bei unbegleiteten minderjährigen Flüchtlingen, S. 3.

4 Dazu Achterfeld Alterseinschätzung bei unbegleiteten minderjährigen Geflüchteten, JAmt 2019, 294; Gelhaar, Die Praxis der Alterseinschätzung von unbegleiteten minderjährigen Flüchtlingen, KJ 2018, 179; Klein, Altersschätzung bei unbegleiteten minderjährigen

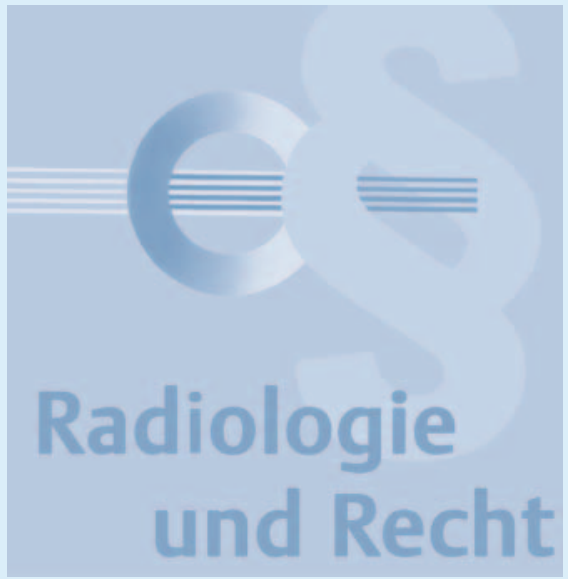

begründeter Zweifel am angegebenen Alter eines Antragstellers oder einer Antragstellerin. Art. 25 Abs. 5 der Verfahrensrichtlinie sieht für Fälle unklaren Alters für die Mitgliedstaaten der EU die Möglichkeit einer ärztlichen Untersuchung zur Altersbestimmung vor. Das BAMF holt vor der Altersfeststellung die Einwilligung der betroffenen Personen und ihres gesetzlichen Vertreters ein.

Das BAMF erwartet außerdem im Zusammenhang mit den medizinischen Gutachten ein auf dem Grundsatz der Verhältnismäßigkeit beruhendes, abgestuftes Verfahren zur Altersfeststellung. Die Durchführung der verschiedenen Untersuchungsschritte soll nach dem Ermessen der durchführenden Ärzte abgeschlossen werden, sobald nach ihrer Einschätzung für die Erstellung eines Altersgutachtens hinreichend gesicherte Erkenntnisse gewonnen worden sind. Die Details zu den einzelnen Untersuchungen sollen den Betroffenen von den Ärzten jeweils vor Ort erläutert und die diesbezügliche Aufklärung sowie die Einwilligung zur Durchführung der einzelnen Maßnahmen im Gutachten dokumentiert werden.

\section{Kritik an den Methoden medizinischer Altersdiagnostik}

In der Vergangenheit wurde unter anderem über den Aussagegehalt der angewandten Methoden der medizinischen Altersdiagnostik, insbesondere über Genauigkeit und Sicherheit der medizinischen Altersfeststellung gestritten. ${ }^{5}$ Da eine exakte medizini-

5 Siehe dazu Schmeling/Dettmeyer/Rudolf/Vieth/ Geserick, a. a. O., S. $113 \mathrm{ff}$. 
sche Ermittlung des Lebensalters nicht möglich ist und dementsprechend das Alter nur geschätzt wird, trifft die medizinische Altersdiagnostik eine Aussage zum wahrscheinlichsten Alter und/oder zu einem bestimmten Mindestalter, so dass im Zweifel - je nach dem in der jeweiligen gesetzlichen Grundlage geforderten Maß der Wahrscheinlichkeit bzw. Sicherheit der Altersaussage - zugunsten des Betroffenen von einem bestimmten Mindestalter, das hinreichend sicher festgestellt werden kann, auszugehen ist. Dieses Mindestalterkonzept stellt sicher, dass das diagnostizierte Lebensalter der begutachteten Person nicht zu hoch angegeben wird, sondern praktisch stets unter dem tatsächlichen Alter liegt. Das OVG Münster stellt in diesem Zusammenhang fest, es liege bereits in der Natur der Sache, dass die medizinische Altersdiagnostik keine exakten Ergebnisse verspreche. Gleichwohl sei nicht zu erkennen, dass die Unsicherheiten bei der ärztlichen Altersfeststellung so weitreichend seien, dass Aufwand und Nutzen in keinem Verhältnis stünden und es aus Sicht des Betroffenen daher von vornherein unverhältnismäßig erschiene, entsprechende Untersuchungen auf sich zu nehmen. ${ }^{6}$

Hinzu kommen in dem Diskurs über medizinische Altersdiagnostik ethische Bedenken sowie rechtliche Zweifel an der Zulässigkeit medizinischer Untersuchungen. So bemängelte der frühere Präsident der Bundesärztekammer, Prof. Dr. Montgomery, vor einigen Jahren zur Praxis des Röntgens der Handwurzelknochen zur Altersbestimmung eine fehlende medizinische Indikation, woraus sich nach seiner Meinung angesichts des mit der Untersuchung einhergehenden Eingriffs in die körperliche Unversehrtheit die Unzulässigkeit dieser Untersuchung ergeben soll. ${ }^{7}$

6 OVG Münster, Beschl. v. 08.09.2015, Az.:12 A 433/15, juris Rn. 12.

7 Siehe dazu die Stellungnahme der Zentralen Kommission zur Wahrung ethischer Grundsätze in der Medizin und ihn Grenzgebieten (Zentrale Ethikkommission) bei der Bundesärztekammer zum Thema „Medizinische Altersschätzung bei unbegleiteten jungen Flüchtlingen“, veröffentlicht u. a. im Deutsches Ärzteblatt, 2016; siehe auch Neundorf, Die Altersbestimmung bei unbegleiteten Minderjährigen - Rechtliche Grundlagen und Debattenüberblick, ZAR 2018, 238, 240.
Die Zentrale Ethikkommission bei der Bundesärztekammer hat noch im Jahre 2016 empfohlen, „bis auf weiteres“ Röntgenund Genitaluntersuchungen zum Zweck der Altersschätzung abzulehnen. Demgegenüber sieht die Arbeitsgemeinschaft für Forensische Altersdiagnostik, bei der es sich - anders als bei der Zentralen Ethikkommission - um den Fachverband der auf dem Gebiet der Altersfeststellung tätigen Wissenschaftler handelt, keine Bedenken an der hinreichenden Aussagekraft medizinischer Untersuchungen zur Altersfeststellung und hält weiterhin angesichts der Geringfügigkeit der Strahlenexposition eine Röntgenuntersuchung für unbedenklich.

\section{Rechtsgrundlagen medizini- scher Altersbestimmungen von Flüchtlingen}

Nach § 24 Abs. 1 S. 1 AsylG ist das BAMF zur Ermittlung des Sachverhalts mit Blick auf das Aufenthaltsrecht zuständig. Allerdings hat das BAMF in der Vergangenheit das Alter von Ausländern nicht selbst bestimmt oder bestimmen lassen, sondern seinen Entscheidungen die Altersbestimmungen anderer Behörden zugrunde gelegt. ${ }^{8}$ Kann das Alter einer möglicherweise noch minderjährigen Person nicht sicher festgestellt werden, richtet sich das behördliche Verfahren zur Altersfeststellung nach $§ 42 \mathrm{f}$. SGB VIII. Das Jugendamt stellt danach im Rahmen der vorläufigen Inobhutnahme der ausländischen Person deren Minderjährigkeit durch Einsicht in eventuell vorhandene Ausweispapiere oder mittels einer qualifizierten Inaugenscheinnahme fest. Auf Antrag des Betroffenen oder von Amts wegen hat das Jugendamt bei verbleibenden Zweifeln eine ärztliche Untersuchung zu veranlassen. Ist eine solche durchzuführen, ist die betroffene Person vom Jugendamt umfassend über die Untersuchungsmethode und über die möglichen Folgen der Altersbestimmung aufzuklären. Ist die ärztliche Untersuchung von Amts wegen durchzuführen, ist die betroffene Person zusätzlich über die Folgen einer Weigerung, sich der ärztlichen Untersuchung zu unterziehen, aufzuklären; die Untersuchung darf

8 Neundorf, ZAR 2018, 238, 241; Antwort der Bundesregierung auf eine Große Anfrage, BT-Drs. 18/5564, S. 72 f. nur mit Einwilligung der betroffenen Person und ihres Vertreters durchgeführt werden. ${ }^{9}$

Allerdings ist eine Altersfeststellung durch das Jugendamt auf Grundlage des $§ 42 \mathrm{f}$. SGB VIII für die Ausländerbehörden nicht bindend. Die Rechtsprechung verweist insoweit auf eine fehlende Tatbestandswirkung des § $42 \mathrm{f}$. SGB VIII, so dass Ausländerbehörden nicht an das Ergebnis einer jugendhilferechtlichen Altersfeststellung gebunden sind. ${ }^{10}$ Insoweit können Ausländerbehörden die Ergebnisse einer solchen Altersbestimmung durch die Jugendämter berücksichtigen; sie sind gleichwohl nicht daran gebunden.

Als weitere Rechtsgrundlage der medizinischen Untersuchung zur Altersfeststellung kommt §49 Abs. 3, Abs. 6 AufenthG in Betracht. Bestehen Zweifel über die Identität, das Lebensalter oder die Staatsangehörigkeit des Ausländers, so sind danach die zur Feststellung seiner Identität, seines Lebensalters oder seiner Staatsangehörigkeit erforderlichen Maßnahmen zu treffen, wenn dem Ausländer die Einreise erlaubt, ein Aufenthaltstitel erteilt oder die Abschiebung ausgesetzt werden soll oder es zur Durchführung anderer Maßnahmen nach dem Aufenthaltsgesetz erforderlich ist. $§ 49$ Abs. 6 AufenthG schreibt dazu ergänzend vor: Maßnahmen im vorgenannten Sinne sind das Aufnehmen von Lichtbildern, das Abnehmen von Fingerabdrücken sowie Messungen und ähnliche Maßnahmen, einschließlich körperlicher Eingriffe, die von einem Arzt nach den Regeln der ärztlichen Kunst zum Zweck der Feststellung des Alters vorgenommen werden, wenn kein Nachteil für die Gesundheit des Ausländers zu befürchten ist. Die Maßnahmen sind zulässig bei Ausländern, die das 14. Lebensjahr vollendet haben; Zweifel an der Vollendung des 14. Lebensjahres gehen dabei zu Lasten des Ausländers. Zur Feststellung der Identität sind diese Maßnahmen nur

9 Dazu Kirchhoff/Rudolf, Altersfeststellung bei unbegleiteten Ausländern vor Inobhutnahmen durch Jugendämter, NVwZ 2017, 1167; Gundelach, Die Rechtsprechung zur medizinischen Altersfeststellung, NVwZ 2018, 1849.

10 Siehe z. B. VGH München, Beschl. v. 23.09.2014, Az.: 12 CE 14.1833, NVwZ-RR 2014, 959; LG Ingolstadt, Beschl. v. 26.07.2018, Az.: 33 T 1074/18, BeckRS 2018, 19238, Rn. $45 \mathrm{ff}$. 
zulässig, wenn die Identität in anderer Weise, insbesondere durch Anfragen bei anderen Behörden nicht oder nicht rechtzeitig oder nur unter erheblichen Schwierigkeiten festgestellt werden kann.

Voraussetzung einer medizinischen Untersuchung zur Altersfeststellung ist demnach, dass diese nach den Regeln der ärztlichen Kunst vorgenommen wird und dass sie nicht die Gesundheit der betroffenen Personen gefährdet. Dazu wird die Ansicht vertreten, dass Röntgenstrahlen ein potentielles Gesundheitsrisiko darstellen, wenngleich die einmalige, kurzzeitige oder auch nur gelegentlich wiederholte ordnungsgemäße Anwendung von Röntgenstrahlen in der Regel nicht als Körperverletzung gewertet wird. ${ }^{11}$

Die Kritik an einer Röntgenuntersuchung stützte sich in der Vergangenheit unter anderem auf die zwischenzeitlich außer Kraft getretene Röntgenverordnung (RöV). Nach § 25 Abs. 1 S. 1 RöV bedurfte der Einsatz von Röntgenstrahlen am Menschen einer medizinischen Indikation oder - wie im Fall der Röntgenuntersuchung im Rahmen der forensischen Altersdiagnostik - einer speziellen gesetzlichen Ermächtigungsgrundlage. Auch bei Vorhandensein einer solchen Ermächtigungsgrundlage sei (zusätzlich) eine rechtfertigende Indikation in jedem Fall erforderlich. ${ }^{12}$ Eine solche medizinische Indikation ergebe sich jedoch nicht aus der aufenthaltsrechtlich erforderlichen Altersbestimmung. ${ }^{13}$ Diese Bedenken sind nicht schon deshalb unberechtigt, weil die RöV zwischenzeitlich außer Kraft getreten ist. Denn auch §119 Strahlenschutzverordnung (StrlSchV) sieht für den medizinischen Einsatz von Röntgenstrahlen eine rechtfertigende medizinische Indikation vor.

11 BGH, Urt. v. 03.12.1997, Az.: 2 StR 397/97, NJW 1998, 833; OVG Hamburg, Beschl. v. 09.02.2011, Az.: 4 Bs 9/11, Beck RS 2011, 47 536; AG Paderborn, Urt. v. 15. März 2019, Az.: 58a C 155/17, juris; dazu Wigge, Keine Körperverletzung durch einmalige Röntgenuntersuchung, RöFo 2019, 873.

13 kritisch insoweit auch Hörich, in: Kluth/Heusch (Hrsg.), BeckOK AusIR, Stand: 01.05.2019, $\S 49$ AufenthG Rn. $34 \mathrm{ff}$.
Von der Rechtsprechung wird diese Kritik an der radiologischen Diagnostik gleichwohl nicht geteilt. Dazu wird zunächst darauf hingewiesen, dass auch radiologische Untersuchungen zur Altersfeststellung mit dem europäischen Unionsrecht vereinbar seien, dieses stehe der Erhebung radiologischer Befunde nicht entgegen. Gemäß Art. 25 Abs. 5 S. 1 der Verfahrensrichtlinie könnten die Mitgliedstaaten im Rahmen der Prüfung eines Antrags auf internationalen Schutz ärztliche Untersuchungen zur Bestimmung des Alters eines unbegleiteten Minderjährigen durchführen lassen, wenn aufgrund allgemeiner Aussagen oder anderer einschlägiger Hinweise Zweifel bezüglich des Alters eines Antragstellers bestehen. Der Begriff „ärztliche Untersuchungen " umfasse dabei auch radiologische Untersuchungen; diese seien nach dem insoweit eindeutigen Wortlaut der Norm nicht ausgeschlossen. ${ }^{14}$ Die Methode, aufgrund einer Kombination einer körperlichen Untersuchung, Röntgenuntersuchungen der Hand und der Zähne sowie einer CT-Untersuchung der Schlüsselbeine auf das Alter zu schließen, sei zur Altersfeststellung geeignet. ${ }^{15}$

Die gutachterlichen Ausführungen eines Sachverständigen müssen nach dem zuletzt zitierten Urteil des OVG Bremen im Hinblick auf Grundlagen, Methodik und Inhalt des Gutachtens den aktuellen Stand der anerkannten wissenschaftlichen Erkenntnis des entsprechenden Fachgebiets widerspiegeln. Ein solcher Stand wissenschaftlicher bzw. vorliegend medizinischer Erkenntnisse liegt - so das OVG Bremen vor, wenn die große Mehrheit der einschlägigen Wissenschaftler und Ärzte die von dem Gutachter herangezogene Methode befürwortet und von einzelnen, nicht ins

14 VG Minden, Urt. v. 13.06.2017, Az.:10 K 240/ 15.A, juris Rn. 40.

15 VG Minden, Urt. v. 13.06.2017, Az.: 10 K 240/ 15.A, juris Rn. 60 unter Verweis auf VGH München, Beschl. v. 05.04.2017, Az.: 12 BV 17.185, juris Rn. 41; OVG Lüneburg, Beschl. v. 22.03.2017, Az.: 4 ME 83/17, juris Rn. 3; OVG Hamburg, Beschl. v. 09.02.2011, Az.: 4 Bs 9/ 11, NordÖR 2013, 72; VG Aachen, Beschl. v. 22.04.2015, Az.: 5 L 15/15.A, juris Rn. 38 ff.; VG Göttingen, Beschl. v. 17.07.2014, Az.: 2 B 195/14, juris Rn. 34 ff.; OVG Bremen, Urt. v. 21.05.2019, Az.: 1 B 86/19, juris; LG Flensburg, Urt. v. 05.02.2019, Az.: I Ks 3/18, juris Rn. 27.
Gewicht fallenden Gegenstimmen abgesehen, über die Methode Konsens besteht. Zusätzlich sei diesbezüglich von Bedeutung, inwieweit sich unter Fachleuten konsensfähige medizinische Erkenntnisse bereits in ärztlichen Leitlinien, Empfehlungen oder Stellungnahmen von Fachgesellschaften niedergeschlagen hätten. ${ }^{16}$ Der aktuelle Stand der anerkannten wissenschaftlichen Erkenntnis auf dem Gebiet der forensischen Altersdiagnostik ergebe sich aus den aktualisierten Empfehlungen für Altersschätzungen der AGFAD und dementsprechend gerade nicht aus den Empfehlungen der Zentralen Ethikkommission bei der Bundesärztekammer. ${ }^{17}$

\section{Fazit}

Die Rechtsprechung hat die Ansicht der Bundesregierung, ${ }^{18}$ dass zu den zulässigen medizinischen Methoden auch die Röntgendiagnostik zählt, bestätigt. Entscheidend ist, dass die ärztliche Untersuchung mit den schonendsten und soweit möglich zuverlässigsten Methoden von qualifizierten medizinischen Fachkräften durchgeführt wird und dass auf den Einsatz der Röntgendiagnostik dort verzichtet wird, wo auch ohne diese Untersuchungsmethode zuverlässige Erkenntnisse über das Alter gewonnen werden können.

Prof. Dr. Martin Beckmann Fachanwalt für Verwaltungsrecht Baumeister Rechtsanwälte Münster

16 OVG Bremen, Urt. v. 21.05.2019, Az.: 1 B 86 19, juris Rn. 6; OVG Bremen, Beschl. v. 04.06.2018, Az.: 1 B 53/18, juris m.w.N.

17 OVG Bremen, Urt.v. 21.05.2019, Az.: 1 B 86/ 19, juris Rn. 6; OVG Bremen, Beschl. v. 04.06.2018, Az.: 1 B 82/18 Rn. 26; OLG Hamm, Beschl. v. 23.10.2018, Az.: 9 UF 104/ 18, Rn. 36; OVG Münster, Beschl.v. 08.09.2015, Az.: 12 A 433/15, juris Rn. 10, 12; zustimmend Kirchhoff/Rudolf, Altersfeststellung bei unbegleiteten Ausländern vor Inobhutnahmen durch Jugendämter, NVwZ 2017, 1167; kritisch dazu Gundelach, die Rechtsprechung zur medizinischen Altersfeststellung eine Anmerkung, NVwZ 2018, 1849.

18 Antwort der Bundesregierung auf eine Kleine Anfrage vom 26.02.2018, BT-Drs. 19/918, S. 3. 